EPJ Web of Conferences 87,04005 (2015)

DOI: $10.1051 /$ epjconf/ 20158704005

(C) Owned by the authors, published by EDP Sciences, 2015

\title{
Remote-Steering Launchers for the ECRH system on the Stellarator W7-X
}

\author{
W. Kasparek ${ }^{1, \mathrm{a}}$, C. Lechte ${ }^{1}$, B. Plaum ${ }^{1}$, A. Zeitler ${ }^{1}$, V. Erckmann ${ }^{2}$, H.P. Laqua ${ }^{2}$, \\ M. Weißgerber ${ }^{2}$, A. Bechtold ${ }^{3}$, M. Busch ${ }^{4}$, B. Szcepaniak ${ }^{4}$ \\ ${ }^{1}$ Institut für Grenzflächenverfahrenstechnik und Plasmatechnologie, Univ. Stuttgart, D-70569 Stuttgart, Germany \\ ${ }^{2}$ Max-Planck-Institut für Plasmaphysik, D-85748 Garching, and D-17491 Greifswald, Germany \\ ${ }^{3}$ NTG Neue Technologie GmbH \& Co KG, D-63571 Gelnhausen, Germany \\ ${ }^{4}$ Galvano-T electroplating-electroforming GmbH, D-51570 Windeck-Rosbach, Germany
}

\begin{abstract}
For electron cyclotron resonance heating of the stellarator W7-X at IPP Greifswald, a $140 \mathrm{GHz} / 10$ MW cw millimeter wave system is in construction. Two out of 12 launchers will employ a remote-steering design. This paper describes the overall design of the two launchers, and design issues like input coupling structures, manufacturing of corrugated waveguides, optimization of the steering range, integration of vacuum windows, mitrebends and vacuum valves into the launchers, as well as tests of prototype parts.
\end{abstract}

\section{Introduction}

At the stellarator Wendelstein7-X (W7-X), an electron cyclotron resonance heating (ECRH) system is under construction. It consists of 10 gyrotrons operating at 140 $\mathrm{GHz}$, with power up to $1 \mathrm{MW} \mathrm{cw}$ each. The transmission from the tubes to the plasma is performed optically via two multi-beam waveguides [1]. The power is injected into the plasma via 10 front-steering launchers, consisting of a focusing reflector and a 2-axes movable plane reflector each. Two switches in the transmission system allow the redirection of the power from two sources to antenna ports at toroidal positions, where the study of trapped-particle physics and quasi-high-field-side launch is possible [2]. These narrow ports do not allow the installation of movable mirrors; therefore, two remotesteering launchers (RSLs) are under construction, which are to be installed in module 1 (RSL1) and module 5 (RSL5), respectively.

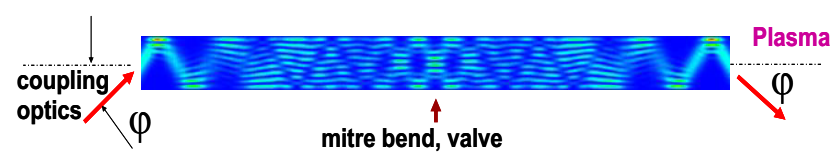

Figure 1. Application of the Talbot effect for remote-steering launchers. The calculation gives the e.m. field in the midplane of the waveguide and illustrates how the field distribution at the waveguide entrance (= input beam) is imaged to the output near to the plasma. Note the minimum of the wall field in the mid of the waveguide.

The remote-steering properties $[3,4,5]$ are based on multimode interference in a corrugated square waveguide leading to imaging effects via the Talbot effect: For a proper length/width of the waveguide, a microwave beam at the input of the waveguide (with a defined direction set by a mirror outside of the plasma vacuum) will exit the waveguide (near the plasma) in the same direction. This is illustrated in Fig. 1.

Remote-steering launchers are suitable for high transmission powers in the MW region $[6,7,8]$. However, the imaging quality decreases the larger the steering angle of the beam is; measures to improve the steering range are going on.

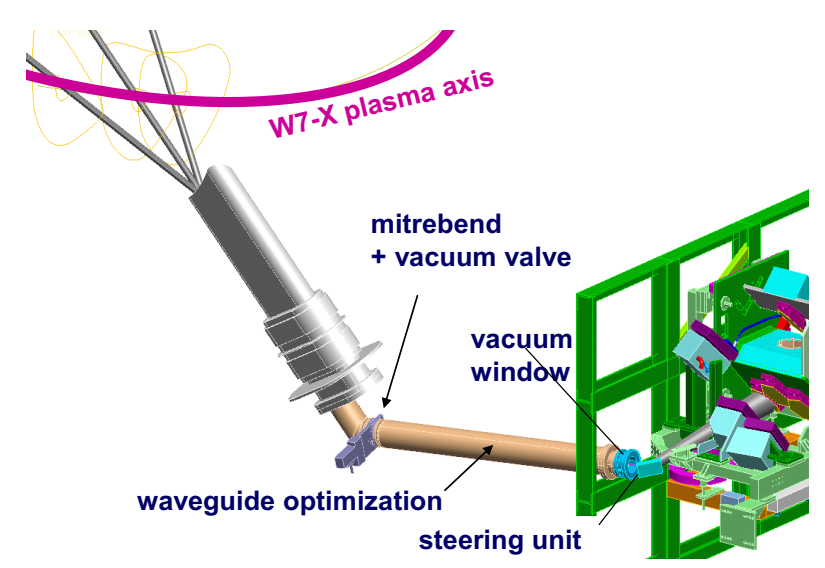

Figure 2. Conceptual design of the remote-steering launcher RSL1 showing the input beam from the optical transmission system (right), the steering unit, vacuum window, mitre bend, and vacuum valve as well as the injection through a port into the plasma, with 3 different beams marking the steering range.

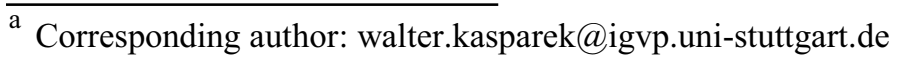


The basic RSL design for W7-X includes the input optics for injection of the beam into the waveguide via a steerable mirror, the vacuum window, and the launcher waveguide ending in front of the plasma. The conceptual design for RSL1 is shown in Fig. 2.

A mitre bend is included in the RSLs to ease integration. The vacuum valve is placed half-way in the waveguide, where the wall fields of the waveguides are low (Fig. 1). In the following, design and integration for the waveguides, mitre bends, vacuum valves and support structures as well as optimization to increase the steering range for the two RSLs, are discussed.

\section{Design of the corrugated square waveguide}

The heart piece of the RSLs is corrugated square waveguide. To fulfil the imaging characteristics for a given wavelength $\lambda$ (and at a steering angle $\varphi$ ), the relation between the length of the waveguide $L_{\text {opt }}$ and its transverse dimension is determined by [9]

$$
L_{\text {opt }}=\frac{4 a^{2}}{\lambda} \cdot \frac{1}{1+0.5 \cdot \sin ^{2} \varphi}
$$

For the design chosen for W7-X, where the length of RSL1 and RSL 5 are $L_{1}=4.6 \mathrm{~m}$ and $L_{5}=5.2 \mathrm{~m}$, and optimization is performed for a steering angle of $\varphi_{\mathrm{opt}}=$ 12.7 degrees ( $c f$. ch. 3), the nominal widths of the waveguides are $a_{1}=50.22$ and $a_{5}=53.4 \mathrm{~mm}$, respectively.

As - in contrast to standard corrugated guides used for transmission - the RSL waveguides are operated at larger Brillouin angles, ohmic absorption is an issue. Therefore, an optimization of the corrugation profile to minimize ohmic loss and birefringence was performed $[10,11]$. Nevertheless, for the quasi-cw operation envisaged, cooling is mandatory.
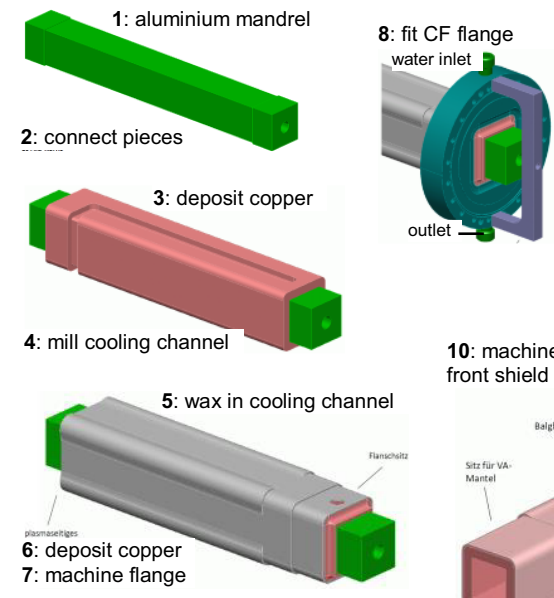

9: deposit coppe on both sides to fix and seal the flange

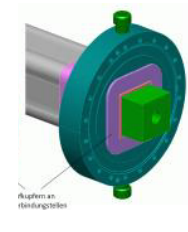

10: machine fittings for bellows, front shield

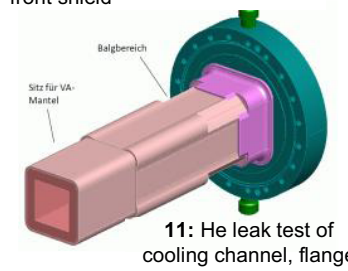

Figure 3. Fabrication steps for the water-cooled, vacuum-tight square corrugated waveguide. The length is not shown to scale. Details see text.
Moreover, the launcher is operated under the plasma vacuum, therefore, high-vacuum performance is required.

These requirements led to the decision to manufacture the waveguides by electroforming from copper. Fig. 3 shows the manufacture steps. Aluminium mandrels with the negative of the corrugation profile are produced in 0.5 $\mathrm{m}$ long pieces; they are joined together for up to $2.5 \mathrm{~m}$ length. Copper is deposited by electroforming. When sufficient thickness is reached, the cooling channels are machined. After filling the cooling channels with wax, electroplating continues until the final wall thickness is reached. The outer contours and especially the connections for the waveguide flanges are machined. Then, special ConFlat vacuum flanges, which also contain water connections, are mounted; in a further process, copper is deposited to both sides of the flange to make the waveguide water- and vacuum-tight. After final machining, removal of the Aluminium mandrels, and $\mathrm{He}$ leak testing, the waveguide is ready.

The fabrication process has been tested by various means, and especially the performance of different groove profiles has been investigated. As best compromise between low ohmic loss, good phase performance, and safe manufacturing, a profile given by Fig. 4 was identified.
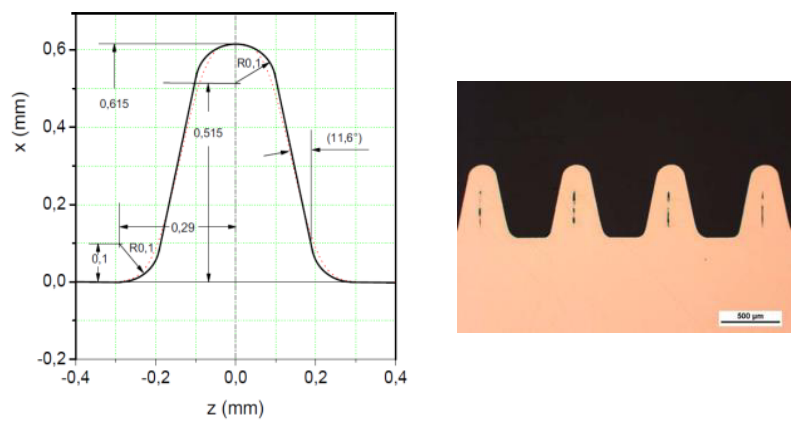

Figure 4. Corrugation profile for the RSL waveguide, $f=140$ $\mathrm{GHz}, p=0.8 \mathrm{~mm}$, and $t=0.615 \mathrm{~mm}$. Left: design drawing; right: grinded cut from an electroformed test sample.

The absorption and the phase shift of the waveguide wall for one reflections was measured in a 3-mirror resonator set up, where the corrugated sample under test forms the 3rd mirror of an open resonator [12,13]. From the Qfactor, the reflection loss can be inferred; the frequencies of the resonances for TE- (E parallel to the grooves) and TM- (E perpendicular to the grooves) polarisation allow the determination of the phase shift.

Note that the total ohmic loss of the waveguide can be calculated to a good approximation by multiplying the single-reflection loss with the number $\mathrm{N}$ of geometrically calculated reflections of the beam, i.e. $\mathrm{N}=\mathrm{L} /(\mathrm{a} \tan \varphi)$ [7]. The same holds for the birefringence of the waveguide, which stems from the summation of the single-reflection phase errors occurring upon each reflection from the waveguide wall. 


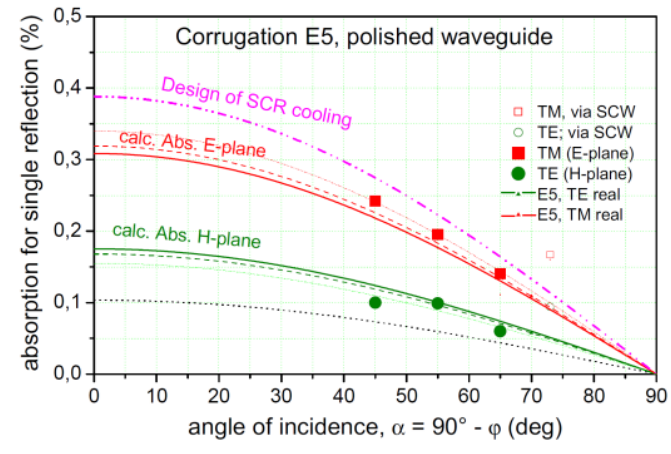

Figure 5. Measured reflection loss for a corrugated waveguide wall with profile E5 (Fig. 4) as function of angle of incidence and polarisation. For reference, the calculated absorption (red and green solid lines), and the assumption for the cooling design (magenta dash dotted line) is plotted.

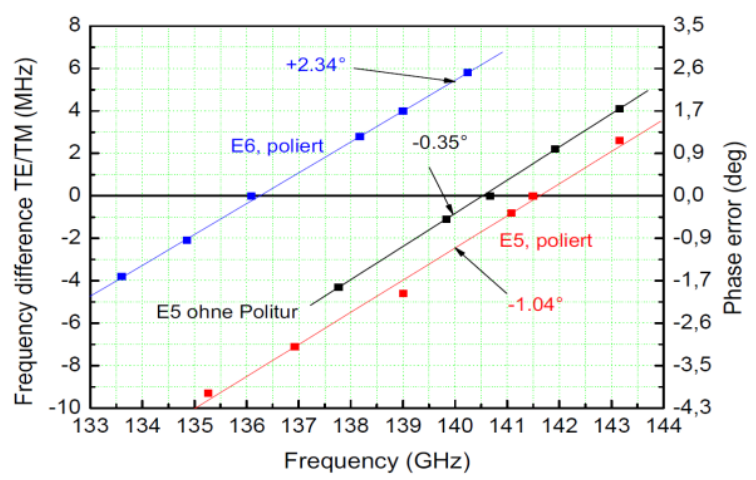

Figure 6. Phase shift of corrugation profile E5 measured at $\varphi=$ $17^{\circ}$, showing low phase errors at $140 \mathrm{GHz}$. The data for an unpolished (black dots) and for a polished (red dots) sample show the influence of electro-polishing of the waveguide wall. Data for a different corrugation profile are shown as well to see typical errors in manufacturing of corrugation profiles.

A worst-case estimation for attenuation and polarisation change for the present RSL5 waveguide with polished surface, operated in TM-polarisation at $\varphi=17^{\circ}$, yields a total ohmic loss of $3 \%$, and a phase shift between the TE and TM polarisation of $31 \mathrm{deg}$.

\section{Design of RSL1}

\subsection{Optimization of steering range}

To reach a high steering range of the launcher, one has to match the effective length of the waveguide such that equation (1) is fulfilled for a broad angular range. Therefore, the movement of the steering mirror is optimized such that the effective waveguide length, i.e. the distance between the beam intersection with the waveguide axis at input and output, is matched to the angle $\varphi$ of the input and output beams $[9,14]$. In Fig. 2, the Gaussian beam efficiency as function of $\varphi$ (for the nominal antenna beam) is plotted for an optimized input coupling with the pivot point moving as function of the steering angle, as well as for a beam which rotates around a fixed pivot point located in the aperture of the waveguide. One can clearly see that an extension of the steering range of $-15^{\circ}<\varphi<15^{\circ}$ wrt. the waveguide axis is reached.

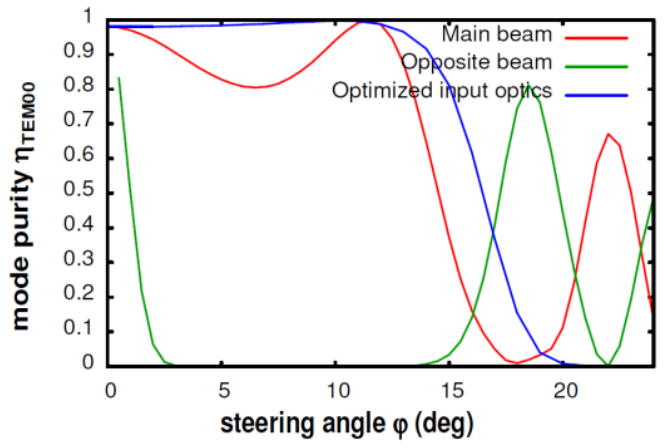

Figure 7. Gaussian beam efficiency for RSL1, when the input beam rotates around a fixed pivot point in the aperture of the waveguide (red), and for a moving pivot point optimized for maximum steering range.

\subsection{Engineering design of RSL1}

The engineering design of RSL 1 at W7-X is shown in Fig. 8. From the multi-beam transmission, one beam is coupled via a steering unit and a diamond vacuum barrier window into the square corrugated waveguide. A linear drive together with a motion link performs a movement of the coupling mirror to get the optimum coupling corresponding to the calculation in Fig. 7. Moreover, the drive can be set such that the "revival" beam at $\varphi=22 \mathrm{deg}$ can be launched.

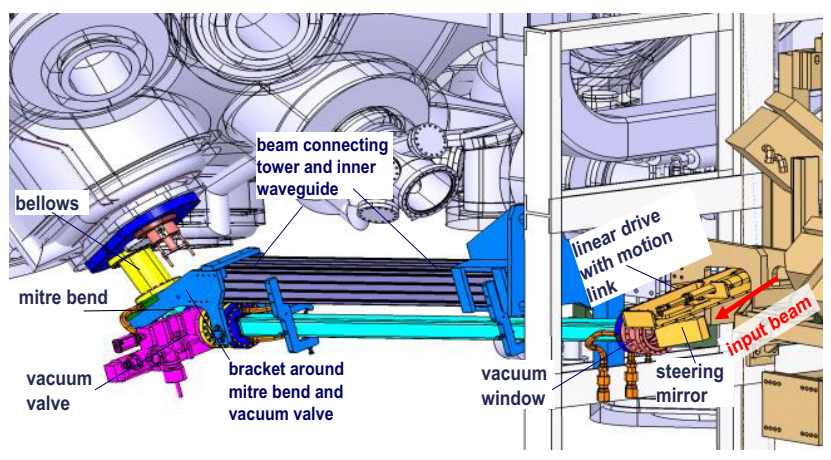

Figure 8. Outline of RSL1, showing the input coupling mirror with mirror drive and motion link, vacuum window, the waveguide with vacuum valve and mitre bend, and the beam connected to the "tower" (right) which supports the complete RSL1.

To fit the RSL1 into the available space at W7-X, a mitre bend is inserted into the waveguide run. For optimum performance of the bend we use a continuous rotation of the grooves with matched depth, as discussed in [15]. Moreover, a gap of $24 \mathrm{~mm}$ length is needed to accommodate a vacuum valve. Note that the incorporation of the valve between vacuum window and waveguide entrance would lead to severe truncation of 
the beam at larger steering angles. With the optimized beam steering, the minimum of the wall field in the RSL waveguide is always exactly half-way in the waveguide run, independent of the steering angle; with further optimization of the gap, low transmission loss and leakage to the valve mechanism is obtained as seen in Fig. 9. Thus a safe operation range of $-15^{\circ}<\varphi<15^{\circ}$ could be reached. Note that the strong coupling to the waveguide gap around $18 \mathrm{deg}$ is the reason, that the steering range around $18 \mathrm{deg}$ using the first revival of the beam ( $c f$. Fig. 7) cannot be used.

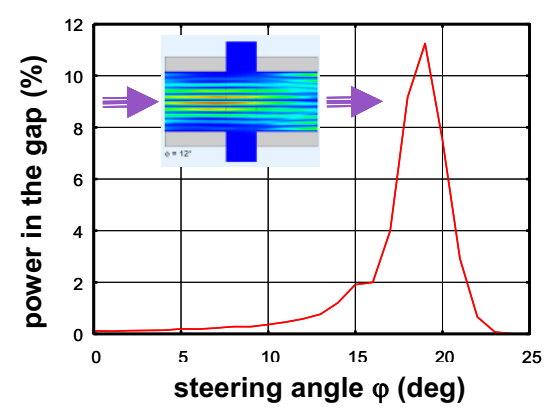

Figure 9. Power radiated into the gap for the waveguide valve as function of the steering angle. Note that the calculation gives the worst case, as further reduction is expected from a reflecting grating structure on the walls of the gap.

All elements of RSL1 are supported by a stiff beam, which is mounted at the "ECRH tower 1" containing the main transmission in front of $\mathrm{W} 7-\mathrm{X}$. The main component is a bracket around the valve and the mitre bend, which takes the main load of the RSL1 and simultaneously avoids a too high bending moment on the valve. Short bellows between the valve and the outer leg of the waveguide is needed for this purpose. The supporting beam can be adjusted such that a possible tilt at the waveguide gap is avoided. The movements of the torus with respect to the RSL structure are compensated by bellows between waveguide and port flange. Except for these bellows, the waveguide part inside the port is not connected to the torus.

\section{DESIGN OF RSL5}

\subsection{Optimization of the steering range.}

The second RSL will be quite similar to the first one, but the steering performance will be further improved by using an optimized waveguide cross-section [16,17]. The dispersion relation of the regular quadratic cross section deviates from the perfect imaging properties at large mode numbers. As a result, the high modes necessary to describe a beam with a large angle have the wrong phase at the waveguide output. By deforming the waveguide cross section, an attempt is made to change the apparent waveguide size for the higher modes, without destroying the good imaging properties of the lower modes.
At present, the properties of nearly square waveguides using various types of deformations are investigated. An example of a waveguide cross-section is shown in Fig. 10, top. The spectrum of the eigenmodes (example in Fig. 10) and their transverse wavevectors is calculated. The Gaussian input beam is decomposed into these eigenmodes, and is propagated through the waveguide. At the output, a beam mode analysis using the parameters of the input beam is performed. The result of such a calculation, as function of the steering angle, is shown in Fig.10 (bottom).
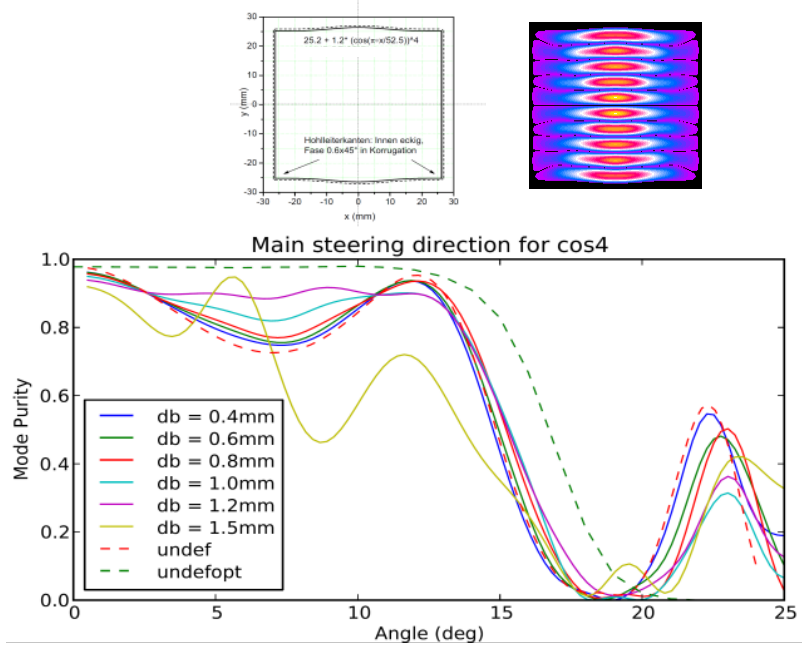

Figure 10. Gaussian beam efficiency for RSL5 for various heights (db) of the bulges as described by eq. (2). The calculation is done for the standard case, where the input beam rotates around a fixed pivot point in the aperture. For comparison, the performance of an undeformed waveguide with fixed beam pivot (red, dashed), and for an undeformed waveguide with optimized input coupling (green, dashed) is plotted.

To this end, a deformation of the waveguide with bulges on both walls described by

$$
\Delta a=1.2 \cdot \cos ^{4}(\pi x / 52.8)
$$

gives the best performance. The results given in [16] could not be fully reproduced; the reason for this is not known. Moreover, the curvature of the bulges is limited by manufacturing constraints; therefore, the possible deformation is only $1 \mathrm{~mm}$. For this reason, the design of RSL5 will employ a combination of deformed waveguide and optimization of the input coupler to get a maximum steering range.

\subsection{Engineering Design}

The engineering design of RSL5 is shown in Fig.11. In contrast to RSL1, where a stiff beam reaching from the tower to the mitre bend takes the mechanical forces, RSL5 is supported mainly at the port flange using a kind of universal joint in conjunction with bellows; in addition, a bracket, which allows some sliding of the waveguide, guides the RSL5 near to the waveguide entrance. 


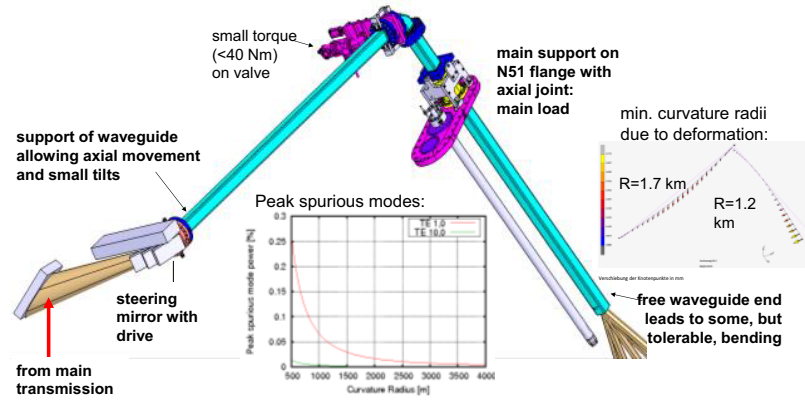

Figure 11. Engineering design of RSL5, showing steering mirror drive, window, waveguide bend, vacuum valve, the support at the N51 port flange and the free waveguide end reaching up to the plasma. The inserts show the deformation of the waveguides due to its own weight with maximum bending radii of 1.7 and $1.2 \mathrm{~km}$, respectively, and the resulting negligible - mode conversion.

Thus, a support with minimum stress on the components is reached. The bending of the waveguide parts inside and outside of the vacuum with minimum radii of $1.7 \mathrm{~km}$ and $1.2 \mathrm{~km}$, respectively, can be tolerated, as can be seen from Fig. 11. The inserts in this figure show the deformation and the resulting mode conversion for two representative modes; a negligible power loss due to mode conversion is expected.

\section{Summary}

Design and construction of two remote-steering launchers for the stellarator $\mathrm{W} 7-\mathrm{X}$ is well underway. The two antennas feature steering mainly in the toroidal plane, and will enhance the experimental flexibility of W7-X. The antennas will be operated under torus vacuum, and the water-cooled design allows cw operation. Therefore, the project is also seen as a technology demonstrator for future applications in fusion reactors [18].

Fabrication of coupling mirrors, waveguide parts and auxiliary components is ongoing. It is expected, to have the first launcher ready for test by end of this year. The installation into W7-X is planned for the opening in 2016.

\section{Acknowledgement}

This work is supported by the German Ministry of Education and Research (BMBF) by grant 03FUS0017A.

\section{References}

1. V. Erckmann et al., Electron cyclotron heating for W7-X: Physics and Technology. Fusion Science and Technology 52, No2 (2007) 291-312.

2. N. Marushchenko et al., this conference.

3. W.H.F. Talbot, Facts relating to optical sciences. No. IV. Philos. Mag. 9 (Third series), 401 (1836).
4. R. Prater, et al., 1997, "The ECH launcher for ITER", Proc. of the 10th workshop on ECE and ECRH, EC-10, ed. T. Donne and T. Verhoeven, World Scientific, Singapore, 531-540 (1997).

5. A.V. Chirkov, et al., Simulation and experimental study of a remote steering system for ECRH/ECCD antenna beams. Fusion Eng. Design 53 (2001) 465 474, 2001.

6. K. Takahashi, C. Moeller, et al., High power experiments of remote steering launcher for electron cyclotron heating and current drive, Fusion Eng. Des. 65 (2003) 589-598.

7. B. Plaum, et al., High-power tests of a remotesteering antenna at $140 \mathrm{GHz}$. Fusion Science and Technology, 50 1-14, 2006.

8. H. Idei, et al., Electron cyclotron current drive experiments in LHCD plasmas using a remote steering antenna on the TRIAM-1M tokamak, Nucl. Fusion 46 (2006) 489-499.

9. K. Ohkubo et al., "Extension of steering angle in a square corrugated waveguide antenna". Fusion Eng. Des. 65 (2003), 657 - 672 .

10. B. Plaum et al., "Numerical calculation of reflection characteristics of grooved surfaces". J. Infrared Milli. Terahz. Waves 32 (2011), 482-495.

11. G.G. Denisov, S.V. Kuzikov, and N. Kobayashi, RF analysis of ITER remote steering antenna for electron-cyclotron plasma heating Int. J. Infrared Millim. Waves 22 (2001) 1735-60.

12. W. Kasparek, A. Fernandez, F. Hollmann, and R. Wacker: Measurement of ohmic loss of metallic reflectors at $140 \mathrm{GHz}$ by a 3-mirror resonator technique. Int. J. Infrared and Millim. Waves 22 (2001) 1695-1707.

13. W. Kasparek, et al., 3-Mirror Resonator Reflectivity Measurement of Plane and Grooved Surfaces: Setup, Options, Results. Proc. of 6th European Conf. on Antennas and Propagation (EU-CAP), Prague, 2012. talk CM01.6, paper 1569533861.

14. W.A. Bongers, et al., A remotely steered millimetre wave launcher for electron cyclotron heating and current drive on ITER. Fusion Eng. Design 85 (2010), pp. $69-86$.

15. W. Kasparek et al., "Performance of a remote steering antenna for ECRH/ECCD applications in ITER using four-wall corrugated square waveguide". Nucl. Fusion 43 (2003), 505 - 1512.

16. G.G. Denisov et al: "Efficiency enhancement of components based on Talbot effect". Int. J. Infrared Milli. Waves 28 (2007), 923-935.

17. C. Lechte et al., Remote-Steering Launchers for the ECRH system on the Stellarator W7-X. Proc. of 38th. Int. Conf. on Infrared, Millimeter and Terahertz waves, IRMMW-THz 2013, Mainz, September 2013, paper A2337676.

18. V. Erckmann, et al., Large Scale CW ECRH Systems: Meeting a Challenge. 19th Topical Conference of RF Power in Plasmas, Vol. 1406 of AIP Conference Proceedings, page 165, Newport, RI, USA, 2011. 